\title{
Biological rhythms and vector insects
}

\author{
Mirian David Marques/+ \\ Museu de Zoologia, Universidade de São Paulo, São Paulo, SP, Brasil
}

The adjustment of all species, animals and plants, to the Earth's cyclic environments is ensured by their temporal organisation. The relationships between parasites, vectors and hosts rely greatly upon the synchronisation of their biological rhythms, especially circadian rhythms. In this short note, parasitic infections by Protozoa and by microfilariae have been chosen as examples of the dependence of successful transmission mechanisms on temporal components.

Key words: temporal organisation - circadian rhythms - Plasmodium - Leishmania - Trypanosoma

This text is a brief survey intended to call attention to the importance of the temporal component in the adjustment of the activity and physiological processes of two interacting species. Although research on biological rhythms is advancing, chronobiological principles and concepts are still rarely used in areas that could benefit from their properties. Therefore, the bibliographic examples cited here have been chosen because their results have been obtained through the use of rigorous chronobiological protocols. These studies are not common and involve a relatively small number of species of parasites and their vectors.

Alternating conditions of light and darkness, warm and cold weather, high and low tides and many other similar phenomena are present in almost all natural environments. Living organisms must adapt to these oscillations and in consequence, their behaviour and physiology must change accordingly. These changes are manifested as different states in biological processes, such as the alternation of food intake and fasting or behaviour, such as activity and rest. They occur in periodic patterns, that is, time intervals that are cyclically repeated and characterising a rhythm. Biological rhythms are observed in almost all known species, from prokaryotes to mammals (Dunlap et al. 2004).

Biological rhythms are not a direct response to a cyclic environment; instead, they are endogenously generated by an oscillator. As such, they are expressed even when the organism is isolated, without access to any environmental cycle, which is known as the free-running condition. The rest/activity rhythm is the best studied biological rhythm and has been described in several species. Its period in the free-running condition is speciesspecific and differs slightly from $24 \mathrm{~h}$, being called, for this reason, "circadian rhythm". The endogenously generated circadian rhythm is adjusted to the precise day

doi: 10.1590/0074-0276130396

+ Corresponding author:mimarque@usp.br

Received 12 August 2013

Accepted 3 December 2013 length by the environmental light/dark (LD) cycle, thus becoming a "daily rhythm". Each species keeps a specific phase-relation to the LD cycle, which is the origin of diurnal, nocturnal and crepuscular species.

Synchronisation entails the establishment of a stable phase relationship between the endogenous rhythm and the environmental cycle and is the primary component of temporal adaptation (Pittendrigh 1981). Many environmental cycles may synchronise circadian rhythms in addition to LD, such as cold/warm and social cycles, as these cycles are repetitive and important to the species (Aschoff 1981).

The circadian system consists of several oscillators that regulate the internal organisation of the organism. The circadian oscillation is generated at the molecular level, as it is the outcome of the expression of several genes that constitute negative feedback loops (Hardin 2011).

Epidemiology and the role of biological rhythms - It is widely accepted that the existence of a circadian system that oscillates in resonance with the environment is adaptive (Yerushalmi \& Green 2009) and temporal adjustments are necessary for survival and reproduction. These adjustments seem to be especially important in the case of parasites [for a detailed review, see Barrozo et al. (2004)]. The parasite life cycle involves different species and the circadian rhythms of the vector and of the host may act as environmental cycles for the parasite. The situation is particularly complex because it implies the coordination of the rhythmic patterns of parasite, vector and host, with the daily cycle of light and darkness (Aschoff 1989). To tackle the problem experimentally is not an easy task given the difficulties of separating the rhythms of the species involved.

Studies on the many species of Plasmodium, on the rhythms of insect vectors and on the symptoms of the host have provided initial information on the subject. Frank Hawking (1970) showed the similarity in the period of the Plasmodium vivax cell cycle and the temperature peaks of infected humans, both of which involved an approximately 48 -h timescale. Results from these studies and those of other Plasmodium species led to the conclusion that Plasmodium life cycles were either $24 \mathrm{~h}$ long or multiples of $24 \mathrm{~h}$ (Hawking et al. 1972). Additionally, it had been demonstrated that Plasmodium knowlesi, 
Plasmodium cynomolgi and Plasmodium cathemerium were capable of infecting mosquitoes only during a restricted time interval of 6-14 h (Hawking et al. 1968). These results suggested that the asexual multiplication of Plasmodium coincided with the time at which vector mosquitoes suck blood (Hawking et al. 1968). This interpretation was confirmed by Garnham and Powers (1974), who called it the "Hawking phenomenon". The Hawking hypothesis, however, implies that the parasites are synchronised among themselves and not necessarily with the vector's rhythms. A clear demonstration that the efficiency of infectivity is due to the fine adjustment between the parasite cell cycle and the rest/activity rhythm of the mammal host was recently achieved by O'Donnell et al. (2011). The authors kept mice in two rooms that were maintained in opposite LD schedules. Mice that were in the dark phase were infected with Plasmodium chabaudi from the room in the light phase and vice-ver$s a$. The results showed a reduction of $50 \%$ in both the in-host replication and the production of transmission stages in the phase-shifted groups when compared with the non-shifted controls, which is evidence of the importance of temporal components in the fitness and survival of malaria parasites.

Although studies involving sandflies are less numerous than those of malaria vectors, a better understanding of the vector's rhythmic activity patterns enhances the understanding of Leishmania transmission. Lutzomyia intermedia and Lutzomyia whitmani, sympatric species that are both vectors of Leishmania braziliensis, which causes cutaneous leishmaniasis, have crepuscular or nocturnal blood-feeding habits. Their activity peaks differ by a short but significant time interval: Lu. intermedia in the nocturne crepuscule and $L u$. whitmani in the middle of the night (Souza et al. 2005). An epidemiological role was attributed to the time difference because $\mathrm{Lu}$. intermedia was collected in a peridomestic area and $L u$. whitmani, in a forest nearby. The interpretation of the effectiveness of both species as vectors of $L$. braziliensis was that in the area studied, Lu. intermedia would be feeding early in the evening and $\mathrm{Lu}$. whitmani would be feeding on early risers, such as agricultural workers (Souza et al. 2005). Interestingly, a similar situation was found in two sibling species of the $\mathrm{Lu}$. longipalpis species complex (Bauzer et al. 2007). Both species, when tested in the laboratory, showed crepuscular activity peaks, with 1-h difference between them and with females more nocturnal than males (Rivas et al. 2008). Again, epidemiological significance was tentatively attributed to the phase differences observed in the two species.

Temporal components are also important in the transmission of the flagellate Trypanosoma cruzi. The Triatominae species that are vectors of the flagellate are all haematophagous species that feed on a diversity of hosts, such as human and domestic animals, as well as other mammals and avian species (Gürtler et al. 2007). A robust activity rhythm has been described in Triatoma infestans: it is expressed as a bimodal rhythm, with a peak at the end of the dark phase and another at the end of the light phase (Lazzari 1992). These two peaks are related to feeding and to the search for refuge, respec- tively (Figueiras \& Lazzari 1998). In the refuges, the insects assemble together and they continue assembled along the light phase. It must be emphasised that the start of activity, blood meal and aggregation keep among them clear phase-relationships as the insects leave the refuge at dusk and search for food, they remain outside until dawn, when aggregation occurs, 8-10 h after feeding (Figueiras \& Lazzari 2000). Each of these temporal steps is under tight endocrine control that, in its turn, is regulated by the circadian system (Vafopoulou \& Steel 2005). Synchronisation of the Triatominae circadian oscillator by the day/night cycle is obviously important because of the long time interval necessary to complete the blood meal, which requires a motionless host. In addition to the day/night cycle, the daily temperature cycle has also been shown to play an important role in the synchronisation of the circadian oscillator (Lazzari 1992). Double activity peaks and the different synchronising environmental cycles are temporal properties that confer plasticity to and increase the transmission potential of Triatominae infected with $T$. cruzi.

Rhythmic expressions have been found in the association of many microfilariae species with their vectors and hosts. Numerous filarial parasites species, transmitted by an equally large number of blood-sucking arthropod vectors, infect a diversity of mammal and birds hosts. Such diversity of involved species leads to the assumption of a similar diversity of temporal strategies of transmission mechanisms. The well-known and basic mechanism is that of the accumulation of the microfilariae in the peripheral blood at times that coincide with the biting activity of the vector (Barrozo et al. 2004). However, the rhythmic processes underlying the patterns of the parasite migration inside the host's body are not yet fully understood. Species such as Wuchereria bancrofti and Brugia malayi present a $24 \mathrm{~h}$ rhythm of distribution, apparently controlled by the oxygen tension in the host's blood; during the day, they remain aggregated in the lungs and migrate to the periphery of the body in the middle of the night. They are transmitted by nightbiting mosquitoes (Hawking 1967). In addition to sensitivity to changes in oxygen tension, the activity of other species, such as Loa loa and Monnigofilaria setariosa, seems to depend on the rhythm of the body temperature of the host as well (Hawking et al. 1967). L. loa is present in peripheral vessels around noon and transmitted by the diurnal Diptera, Chrysops (Duke 1960). The host's oxygen tension and temperature cycle seem to be the primary synchronising factors of the parasite activity rhythm. However, the existence of a Pacific strain of $W$. bancrofti whose activity is intermediate between diurnal and nocturnal implies a possible plasticity of the filariae processes of synchronisation. This plasticity seems to be extended to the temporal system itself, as can be exemplified by the absence of a rhythm of distribution in Mansonella perstans and Dipetalonema gracile that would adjust the population to the feeding habits of the vectors. The problems of explaining the microfilariae temporal system were initially attributed by Hawking (1967) to the weak endogenous circadian rhythm that an individual worm presents. More recent 
efforts to elucidate which physiological components on the circadian rhythms of the human body could be the synchronising factors of the microfilariae rhythms have also failed (Hawking et al. 1981).

The insects' circadian system - In the examples cited here, the importance of temporal factors in the adjustments of parasites, their vectors and hosts is clear. Several rhythms of different frequencies and amplitudes play central roles in their life cycles, the circadian rhythm being the most relevant.

The insects' circadian system consists of coupled pacemakers, a multioscillatory system. It is composed of peripheral oscillators, found in the integument, Malpighian tubules, antennal chemosensory hairs, sperm ducts and others (Saunders 2002) and a central or master oscillator situated in the central nervous system. Environmental cyclic cues, which are responsible for the synchronisation of the endogenous rhythm, reach the circadian system through afferences, usually sensory pathways. The rhythms generated by the pacemakers are communicated to the entire body through the efferences, most of which are hormones or neurotransmitters.

This organismal structure is paralleled on the molecular scale. The feedback loops harbour dedicated genes that are sensitive to a particular environmental factor, such as cryptochrome, the photoreceptor of Drosophila. The transmission of the rhythm generated by the loops depends on the action of a set of "clock-controlled genes" (Hardin 2011). Results obtained through the study of the molecular oscillator of vectors represent new tools to explain components of the mechanisms of infection (Meireles-Filho et al. 2006), specific functional characteristics (Gentile et al. 2006, 2009) and conditions that increase the infectivity potential (Lima-Camara et al. 2011), among others. Notwithstanding these efforts, a great deal of research on the biological rhythms of vector insects remains to be performed if we want to achieve more effective tools of control. A full description of the molecular oscillators of pathogens may lead to the development of forms with shifted activity peaks, for instance, that would render transmission impossible.

\section{ACKNOWLEDGEMENTS}

To the editors, for the invitation to participate in this special issue, so very special for many reasons, and Alexandre Afranio Peixoto is present in all of them.

\section{REFERENCES}

Aschoff J 1981. Freerunning and entrained circadian rhythms. In J Aschoff (ed.), Handbook of behavioral neurobiology. Biological rhythms, Vol. 4, Plenum Press, New York, p. 81-93.

Aschoff J 1989. Temporal orientation: circadian clocks in animals and humans. Anim Behav 37: 881-896.

Barrozo RB, Schilman PE, Minoli SA, Lazzari CR 2004. Daily rhythms in disease-vector insects. Biol Rhythm Res 35: 79-92.

Bauzer LGSR, Souza NA, Maingon RDC, Peixoto AA 2007. Lutzomyia longipalpis in Brazil: a complex or a single species? A minireview. Mem Inst Oswaldo Cruz 102: 1-12.

Duke BOL 1960. Studies on the biting habits of Chrysops. VII. The biting cycles of nulliparous and parous C. silacea and C. dimitiata (Bombe-form). Ann Trop Med Parasitol 54: 147-155.
Dunlap JC, Loros JJ, DeCoursey PJ 2004. Chronobiology: biological timekeeping, Sinauer Associates, Sunderland, 406 pp.

Figueiras ANL, Lazzari CR 1998. Aggregation in the haematophagous bug Triatoma infestans: a novel assembling factor. Physiol Entomol 23: 33-37.

Figueiras ANL, Lazzari CR 2000. Temporal change of the aggregation response in Triatoma infestans. Mem Inst Oswaldo Cruz 95: 889-892.

Garnham PCC, Powers KG 1974. Periodicity of infectivity of plasmodial gametocytes: the "Hawking phenomenon". Int J Parasitol 4: 103-106.

Gentile C, Meireles-Filho ACA, Britto C, Lima JBP, Valle D, Peixoto AA 2006. Cloning and daily expression of the timeless gene in Aedes aegypti (Diptera: Culicidae). Insect Biochem Mol Biol 36: 878-884.

Gentile C, Rivas GBS, Meireles-Filho CA, Lima JBP, Peixoto AA 2009. Circadian expression of clock genes in two mosquito disease vectors: cry2 is different. $J$ Biol Rhythms 24: 444-451.

Gürtler RE, Ceballos LA, Ordóñez-Krasnowski P, Kitron U, Cohen JE 2007. Domestic dogs and cats as sources of Trypanosoma cruzi infection in rural northwestern Argentina. Parasitology 134: 69-82.

Hardin PE 2011. Molecular genetic analysis of circadian timekeeping in Drosophila. Adv Genet 74: 141-173.

Hawking F 1967. The 24-hour periodicity of microfilariae: biological mechanisms responsible for its production and control. Proc Biol Sci 169: 59-76.

Hawking F 1970. The clock of the malaria parasite. Sci Am 222: 123131.

Hawking F, Gammage K, Worms MJ 1972. The asexual and sexual circadian rhythms of Plasmodium vinckei chabaudi of $P$. berghei and of $P$. gallinaceum. Parasitology 65: 189-201.

Hawking F, Jennings T, Louis FJ, Tuira E 1981. The mechanisms which affect the periodic cycle of Pacific Wuchereria bancrofti microfilariae. J Helminthol 55: 95-100.

Hawking F, Moore P, Gammage K, Worms MJ 1967. The effect of variations in host body temperature on the cycle of Loa loa, Monnigofilaria setariosa, Dirofilaria immitis and other filariae. Trans R Soc Trop Med Hyg 61: 674-683.

Hawking F, Worms MJ, Gammage K 1968. 24 and 48-hour cycles of malaria parasites in the blood; their purpose, production and control. Trans R Soc Trop Med Hyg 62: 731-760.

Lazzari CR 1992. Circadian organization of locomotion activity in the haematophagous bug Triatoma infestans. J Insect Physiol 38: 895-903.

Lima-Camara TN, Bruno RV, Luz PM, Castro MG, Lourenço-deOliveira R, Sorgine MHF, Peixoto AA 2011. Dengue infection increases the locomotor activity of Aedes aegypti females. PLoS ONE 6: 1-5.

Meireles-Filho ACA, Rivas GBS, Gesto JSM, Machado RC, Britto C, Souza NA, Peixoto AA 2006. The biological clock of an hematophagous insect: locomotor activity rhythms, circadian expression and downregulation after a blood meal. FEBS Lett 580: 2-8.

O’Donnell AJ, Schneider P, McWatters HG, Reece SE 2011. Fitness costs of disrupting circadian rhythms in malaria parasites. Proc Biol Sci 278: 2429-2436.

Pittendrigh CS 1981. Entrainment. In J Aschoff (ed.), Handbook of behavioral neurobiology. Biological rhythms, Vol. 4, Plenum Press, New York, p. 95-124. 
Rivas GBS, Souza NA, Peixoto AA 2008. Analysis of the activity patterns of two sympatric sandfly siblings of the Lutzomyia longipalpis species complex from Brazil. Med Vet Entomol 22: 288-290.

Saunders DS 2002. Insect cocks, 3rd ed., Elsevier, Amsterdam, 576 pp.

Souza NA, Andrade-Coelho CA, Peixoto AA, Rangel EF 2005. Nocturnal activity rhythms of Lutzomyia intermedia and Lutzomyia whitmani (Diptera: Psychodidae) in a transmission area of Amer- ican cutaneous leishmaniasis in Rio de Janeiro state, Brazil. $J$ Med Entomol 42: 986-992.

Vafopoulou X, Steel CGH 2005. Circadian organization of the endocrine system. In LI Gilbert, K Latrou, SS Gill (eds.), Comprehensive molecular insect science, Vol. 3, Elsevier, Oxford, p. 551-614.

Yerushalmi S, Green RM 2009. Evidence for the adaptive significance of circadian rhythms. Ecol Lett 12: 970-981. 